\title{
Intralesional Bleomycin for Treatment of a Recalcitrant Wart in an Immunocompromised Patient: A Safe, Effective, and Underutilized Therapy
}

\author{
Tracy $\mathrm{Ngo}^{1}$, Hannah Hwang ${ }^{2}$, Bijal Amin ${ }^{2}$, and Steven Cohen ${ }^{1}$ \\ ${ }^{1}$ Albert Einstein College of Medicine \\ ${ }^{2}$ Montefiore Medical Center
}

October 3, 2021

\begin{abstract}
Injectable bleomycin is infrequently used for recalcitrant warts despite its efficacy, acceptable safety profile, and high patient satisfaction compared to other treatment modalities. We present an immunocompromised patient with a large recalcitrant wart successfully treated with intralesional bleomycin to provide greater clinical exposure, training, and practice with intralesional bleomycin.
\end{abstract}

Title: Intralesional Bleomycin for Treatment of a Recalcitrant Wart in an Immunocompromised Patient: A Safe, Effective, and Underutilized Therapy

Authors: Tracy Ngo BS${ }^{1}$, Hannah Hwang DNP², Bijal Amin MD ${ }^{3}$, Steven R. Cohen MD ${ }^{2}$

Affiliation:

1. Albert Einstein College of Medicine, Bronx NY

2. Albert Einstein College of Medicine, Division of Dermatology, Bronx NY

3. Albert Einstein College of Medicine, Department of Pathology, Bronx NY

Key words: Bleomycin, verruca vulgaris, wart, immunocompromised, HIV

Corresponding Author :

Steven R. Cohen

Address :

Einstein-Montefiore Division of Dermatology, Montefiore Medical Center

111 East $210^{\text {th }}$ Street

Bronx, NY 10467

Email :

steven.cohen@einsteinmed.org

Telephone :

(718) 920-2176 
Funding Source : This article has no funding source.

Conflicts of Interest : The authors have no conflict of interest to declare.

Prior presentation : This article has no prior presentation or publication.

Reprint request : steven.cohen@einsteinmed.org

Word Count : 598

References : 8

Figures : 3

Abbreviations used: HIV, human immunodeficiency virus; HAART, highly active antiretroviral therapy

\begin{abstract}
Injectable bleomycin is infrequently used for recalcitrant warts despite its efficacy, acceptable safety profile, and high patient satisfaction compared to other treatment modalities. We present an immunocompromised patient with a large recalcitrant wart successfully treated with intralesional bleomycin to provide greater clinical exposure, training, and practice with intralesional bleomycin.
\end{abstract}

\title{
Key Clinical Message
}

Injectable bleomycin is a potent, safe, and well accepted treatment for recalcitrant warts and its use should be encouraged through greater provider exposure, training, and practice.

\section{Introduction}

Warts are common benign lesions caused by the human papillomavirus which affect approximately $10 \%$ of the global population. ${ }^{1}$ In high-risk populations such as children or immunocompromised individuals, the prevalence may be as high as $20 \%$ percent. ${ }^{1}$ Despite the expanding arsenal of wart therapies, recalcitrant verrucae are quite common, not only in those with predisposing risk factors, but also in many otherwise healthy persons. Intralesional bleomycin is a well-tolerated remedy with proven safety and satisfaction, as well as a track record of high efficacy. ${ }^{2-4}$ Despite decades of experience and data supporting injectable bleomycin for recalcitrant warts, this treatment remains uncommon in clinical practice. We present an immunocompromised patient with a large recalcitrant wart successfully treated with intralesional bleomycin, underscoring the ease and efficacy of this modality, while encouraging its use for verrucae unresponsive to other modalities.

\section{Case Report}

A 71-year-old male with human immunodeficiency virus (HIV) infection on highly active antiretroviral therapy (HAART) with a CD4 count of 534 and an undetectable viral load was referred to dermatology for evaluation of a painful lesion on the left buttock of one-year duration. The lesion had steadily enlarged for six months before growth halted and crusting ensued. On examination, a solitary, firm verrucous plaque on the left buttock measured $1.6 \mathrm{~cm}$ by $2.0 \mathrm{~cm}$ (Fig. 1a). A slice biopsy specimen demonstrated histologic features of verruca vulgaris. The lack of a response topical imiquimod cream, 3.75\%, for two months led to curettage surgery (Fig. 1b).

Recurrence of the verruca within 4-weeks of curettage led to treatment with intralesional bleomycin. The affected area was anesthetized with lidocaine HCL, $2 \%$ plus epinephrine $(1: 100,000)$. Verrucous tissue was pared flat with a \#15 blade, and $0.5 \mathrm{~mL}$ of bleomycin sulfate $(3 \mathrm{U} / \mathrm{mL})$ was injected directly into verrucous foci at a depth of 1 to $1.5 \mathrm{~mm}$. Injection site healed with residual hypopigmentation; no recurrence at 10-weeks (Fig. 1c).

\section{Discussion}


Bleomycin sulfate is a water-soluble compound composed of cytotoxic glycopeptide antibiotics isolated from a strain of Streptomyces verticillus ${ }^{5}$ Use of intralesional bleomycin for treatment of recalcitrant warts has been reported since the late 1970s, with multiple studies showing 59-100\% efficacy with an acceptable safety profile. Transient pain is the most frequent side effect. ${ }^{2}$ Cure rates are lowest for plantar warts (approximately $60 \%$ ), but can be reliably high for periungual and other extremity locations (94\% and $95 \%$, respectively). ${ }^{6}$ It is postulated that keratinocyte apoptosis induced by bleomycin leads to elimination of virus. ${ }^{7}$ Aside from pain, local (e.g., Raynaud's phenomenon) and systemic (e.g., flagellate hyperpigmentation) side effects are rare. ${ }^{2,6}$ Because intralesional bleomycin can lead to increased serum drug levels, a thorough history and counseling is required, especially for women of childbearing potential. ${ }^{2,6,7}$ Plasma bleomycin concentrations may be reduced by using doses as low as $0.25 \mathrm{U} / \mathrm{mL}$ with reported efficacy. ${ }^{8}$

Despite the ease of administration along with decades of documented efficacy and safety, intralesional bleomycin remains a last-resort treatment for warts. ${ }^{3}$ A study by Kruter et al. showed that $78 \%$ of patients reported satisfaction with intralesional bleomycin. ${ }^{2}$ The treatment was recommended by $97 \%$ of those who attained complete remission $(74 \%)$. Given the high efficacy and high rate of satisfaction compared to other destructive modalities, bleomycin is likely underused in clinical practice due to provider inexperience. ${ }^{2,6}$ Following proper injection technique neutralizes risks of transient Raynaud phenomenon when treating warts on digits and/or ulceration from an excessive volume of bleomycin per injection site. ${ }^{2}$

With increased exposure, training, and experience, providers will feel more confident about offering intralesional bleomycin as second- or third-line treatment of warts. Our case underscores that bleomycin is a simple, safe, and effective therapy for recalcitrant warts in nonpregnant or lactating patients.

\section{References}

1. Al Aboud A, Nigam P. Wart. StatPearls: Treasure Island (FL); 2020.

2. Kruter L, Saggar V, Akhavan A, Patel P, Umanoff N, Viola KV, Stebbins W, Smith E, Cohen JV, Cohen SR. Intralesional Bleomycin for Warts: Patient Satisfaction and Treatment Outcomes. J Cutan Med Surg 2015;19(5):470-6.

3. Dall'oglio F, D'Amico V, Nasca MR, Micali G. Treatment of cutaneous warts: an evidence-based review. Am J Clin Dermatol 2012;13(2):73-96.

4. Amer M, Diab N, Ramadan A, Galal A, Salem A. Therapeutic evaluation for intralesional injection of bleomycin sulfate in 143 resistant warts. Journal of the American Academy of Dermatology 1988;18(6):13131316.

5. Shumer SM, O'Keefe EJ. Bleomycin in the treatment of recalcitrant warts. Journal of the American Academy of Dermatology 1983;9(1):91-96.

6. Abess A, Keel DM, Graham BS. Flagellate Hyperpigmentation Following Intralesional Bleomycin Treatment of Verruca Plantaris. Archives of Dermatology 2003;139(3):337-339.

7. James MP, Collier PM, Aherne W, Hardcastle A, Lovegrove S. Histologic, pharmacologic, and immunocytochemical effects of injection of bleomycin into viral warts. Journal of the American Academy of Dermatology 1993;28(6):933-937.

8. Hayes ME, O'Keefe EJ. Reduced dose of bleomycin in the treatment of recalcitrant warts. Journal of the American Academy of Dermatology 1986;15(5):1002-1006.

Figure 1: Verruca vulgaris treated with bleomycin. a) Verruca on left buttock at initial presentation. b) Recurrence of verruca status post slice biopsy and persistence after two months of treatment with imiquimod $3.75 \%$ cream. c) No residual or recurrence of warty activity 5 weeks after treatment with intralesional bleomycin. 

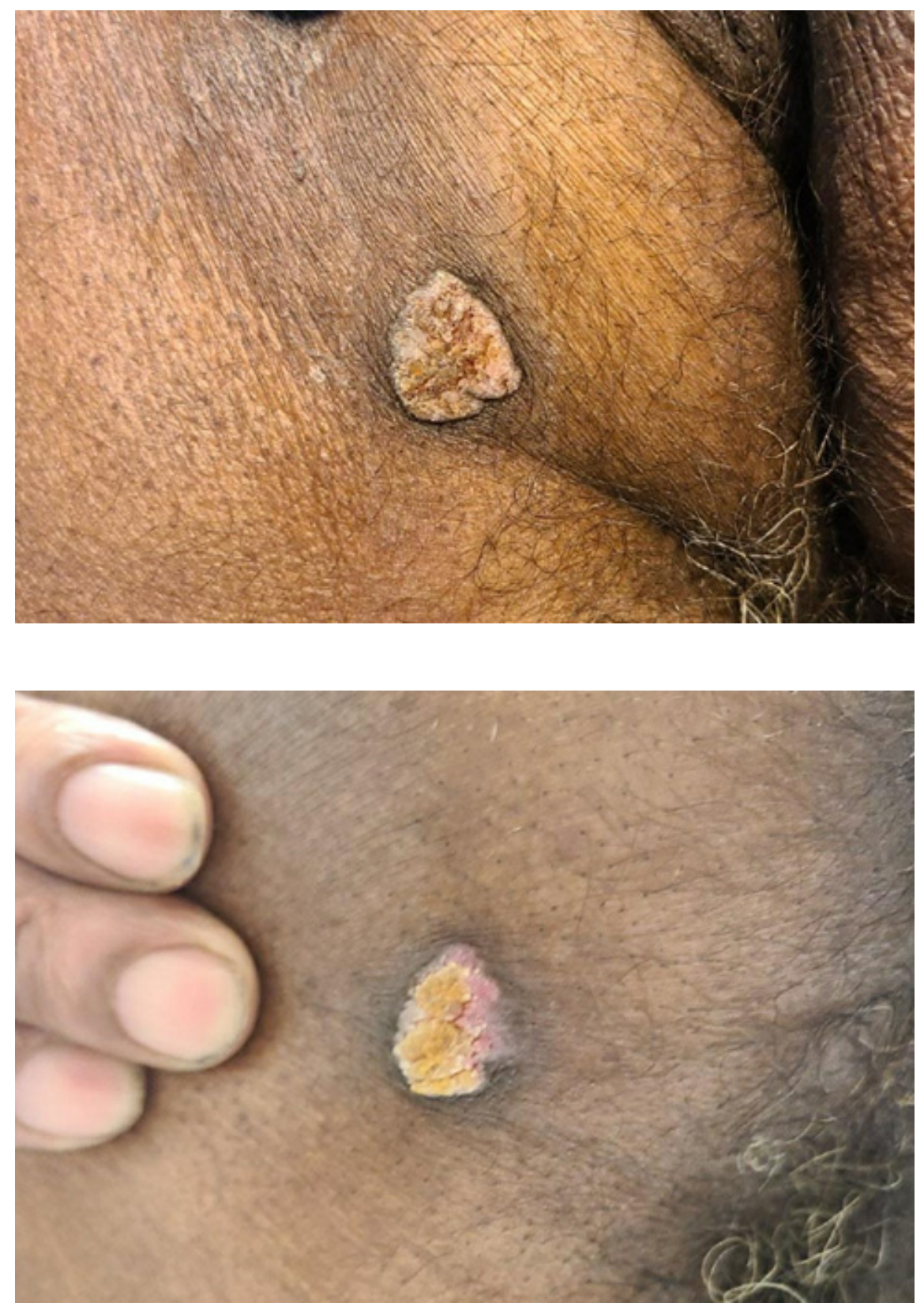


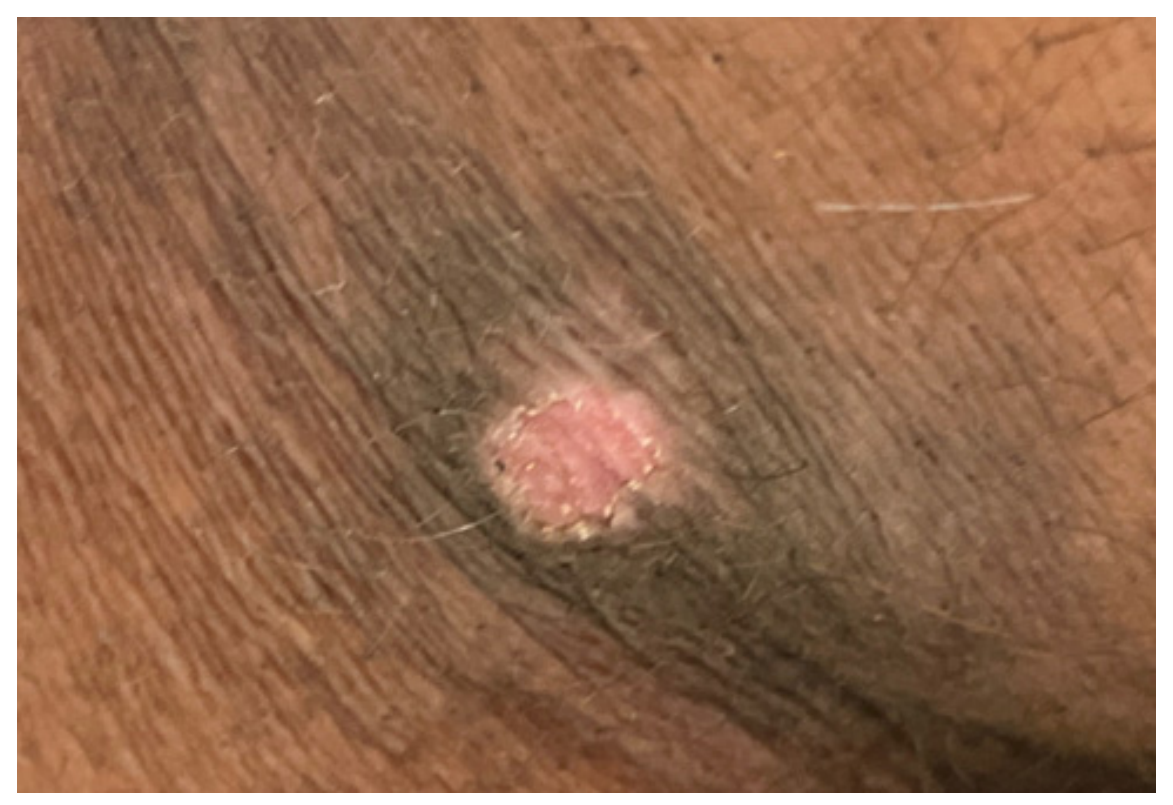

1.

2.

3.

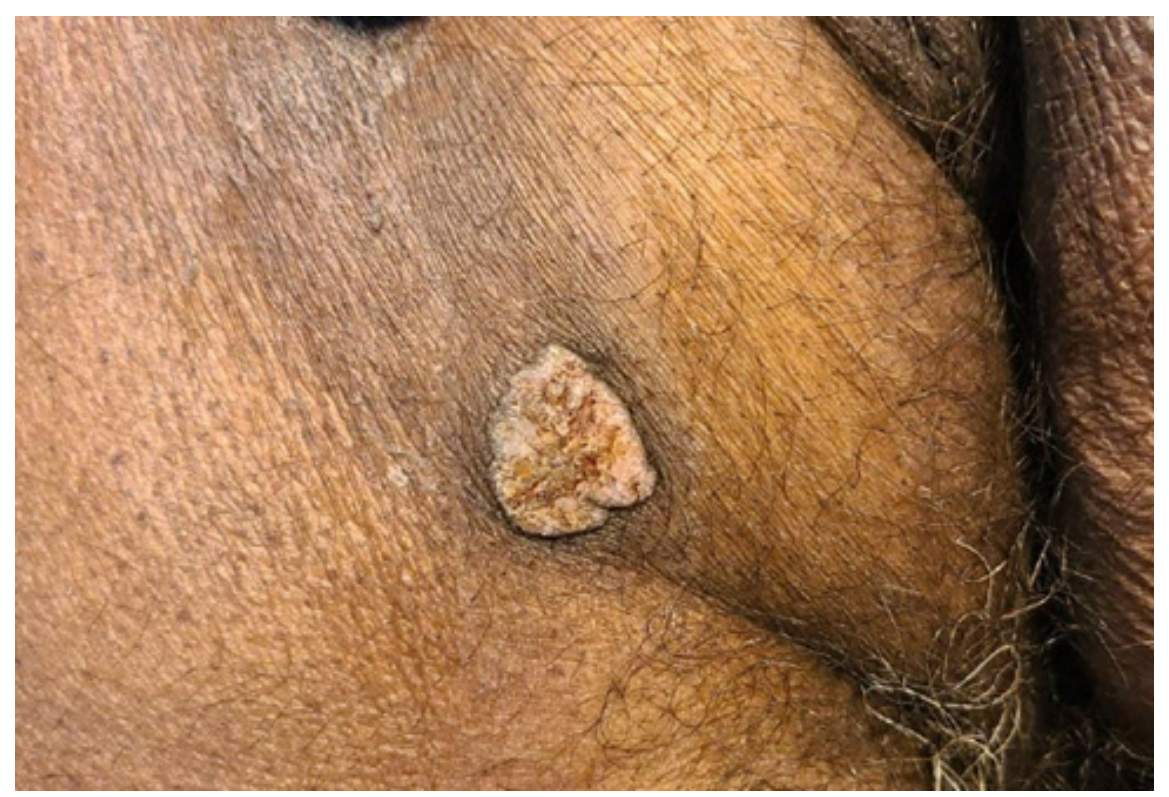



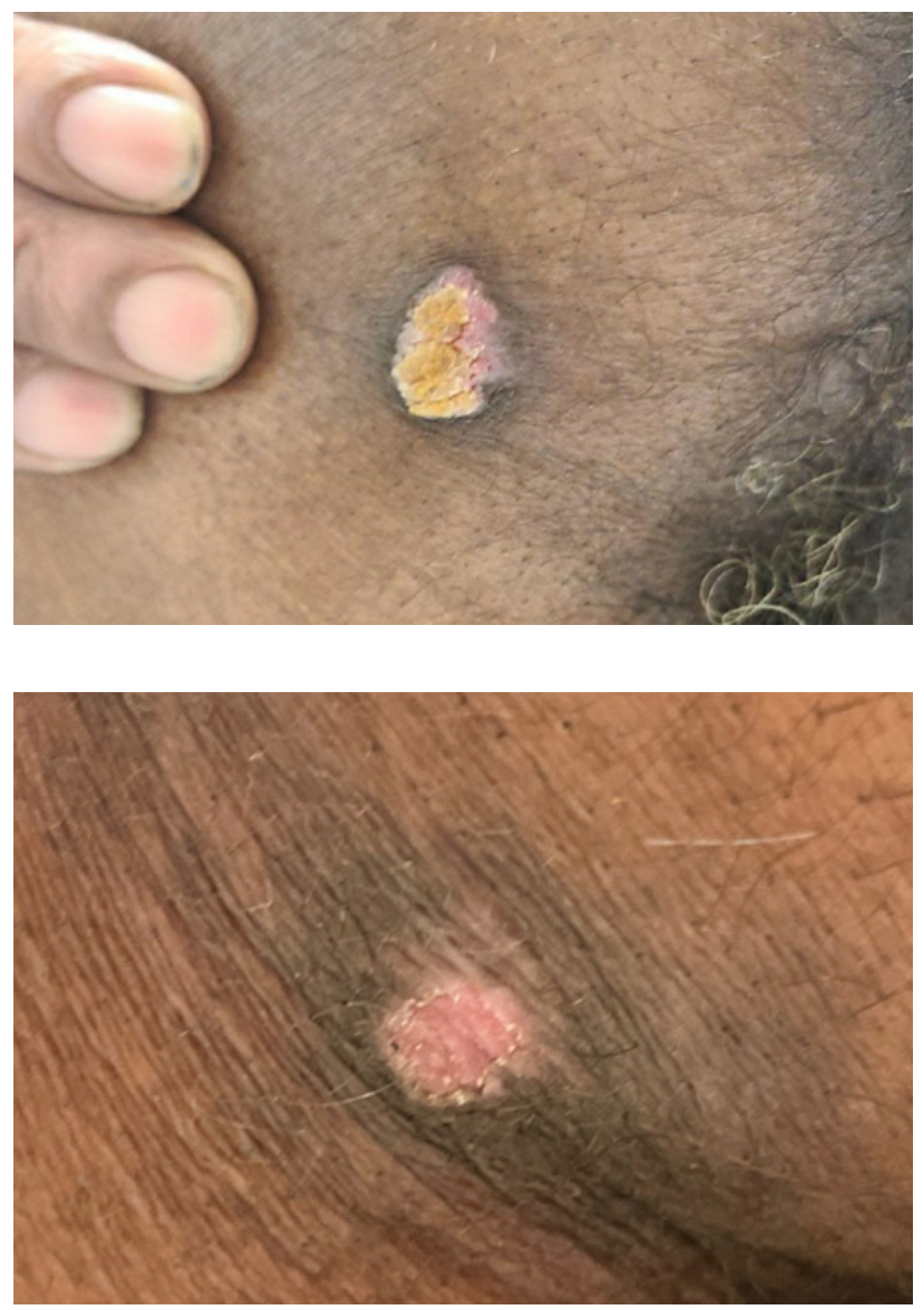